AIR COMMAND AND STAFF COLLEGE

AIR UNIVERSITY

\title{
A Persistent Perch: USSOCOM's Use of Organic Space Based ISR
}

\author{
by \\ Christian P. Helms, Major, United States Air Force
}

A Research Report Submitted to the Faculty

In Partial Fulfillment of the Graduation Requirements for the Degree of

\section{MASTER OF OPERATIONAL ARTS AND SCIENCES}

Advisor: Mr. Michael P. Ivanovsky

Maxwell Air Force Base, Alabama

May 2015 


\section{Disclaimer}

The views expressed in this academic research paper are those of the author and do not reflect the official policy or position of the US government or the Department of Defense. In accordance with Air Force Instruction 51-303, it is not copyrighted, but is the property of the United States government. 


\section{Table of Contents}

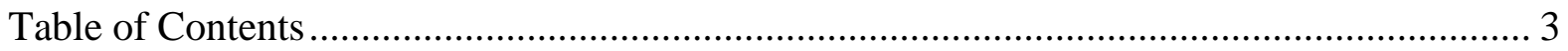

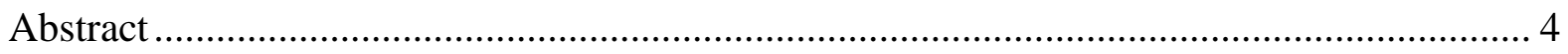

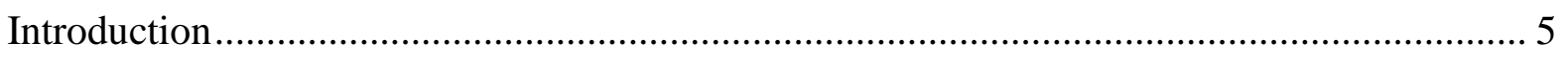

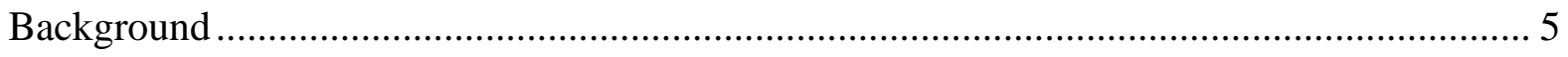

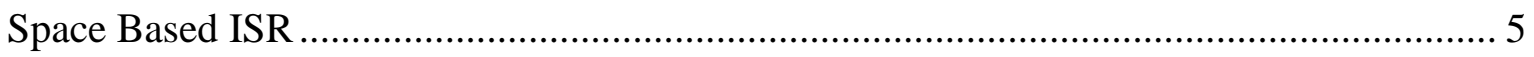

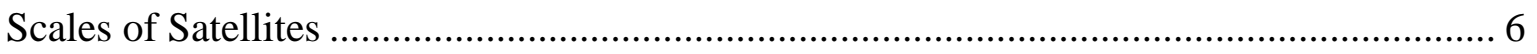

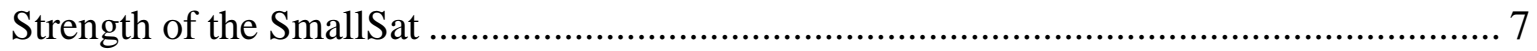

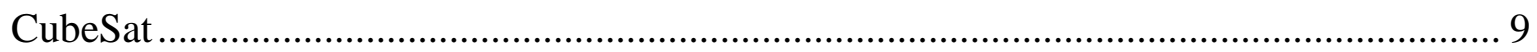

Current Coverage / Demand Disconnects ................................................................ 10

Development of Organic SOF Space ISR Capabilities................................................. 12

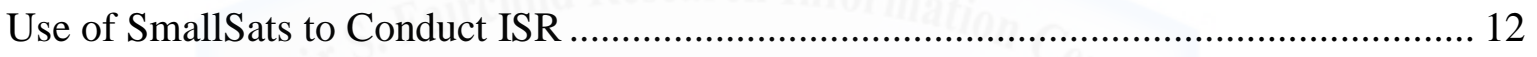

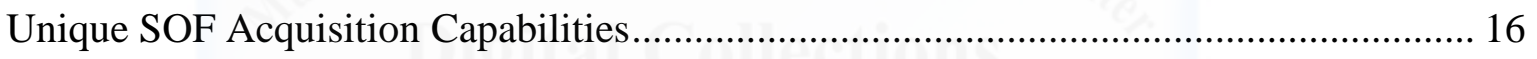

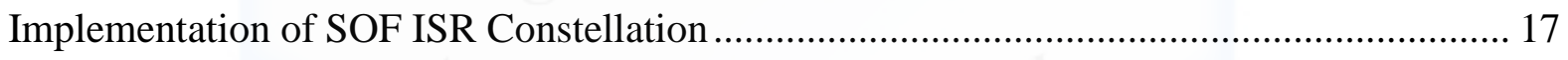

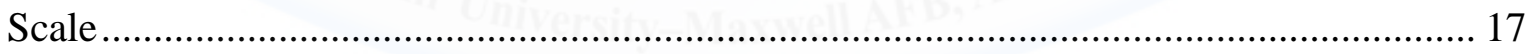

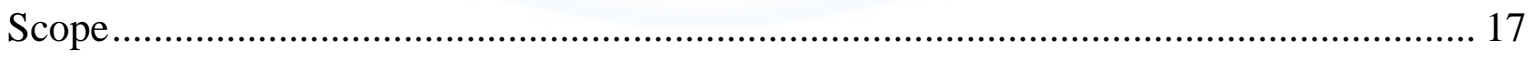

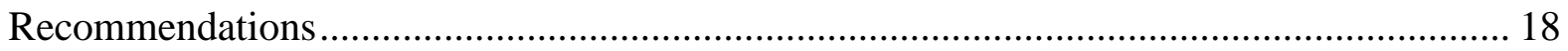

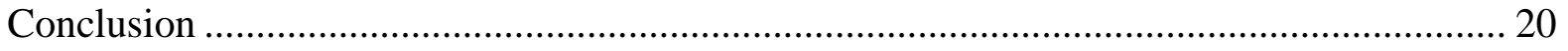

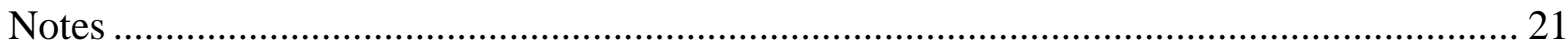

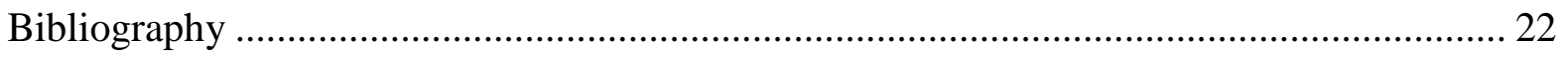




\begin{abstract}
US Special Operations Command (USSOCOM) has, for the better part of the past decade, been America's lead combatant command in the fight against terrorism. SOCOM has a demanding repertoire of activities that require significant support from a variety of different warfighting enablers, most notably intelligence, surveillance, and reconnaissance (ISR) assets. In order to augment and supplement the command's dependence on persistent air-breathing ISR orbits, USSOCOM needs to consider fielding the capability to procure, configure, launch, and control its own constellation of space based ISR satellites. Space based ISR has the potential to provide the command with near real time imagery intelligence (IMINT) over areas of the world that are anti-access or area denied (A2AD). A2AD areas of the globe frustrate SOCOM planners and operators as the feasibility to park an airborne ISR asset over an A2AD environment for weeks on end is neither feasible nor possible. If SOCOM leveraged nascent technologies, like CubeSats and fractionated satellite architecture, the command could procure, launch, and control its own satellite constellations cheaper than traditional satellite systems or airborne ISR vehicles.
\end{abstract}




\section{Introduction}

As US Special Operations Command (USSOCOM) prosecutes its mission as America's lead counterterrorism combatant command, its geographically distributed forces will continue to put a high demand on both the nation's intelligence, surveillance, and reconnaissance (ISR) assets. This demand comes at a time when numerous other military commands and US government agencies are vying to grab critical minutes of ISR asset allocation to support the myriad of US defense activities abroad. In order to remain ahead of our adversary, and maintain on-demand ISR capability, USSOCOM needs to consider fielding the capability to procure, configure, launch, and control its own constellation of space based ISR satellites.

A viable solution to USSOCOM's voracious appetite for ISR products, specifically near-realtime imagery intelligence of an area of interest, is for the command to field its own constellation of ISR satellites tailor made to support its uniquely sensitive and time critical mission sets. Whereas this concept was historically cost prohibitive and technologically unfeasible, burgeoning technologies have enabled the creation of small-scale satellites with large satellite capabilities at a fraction of the cost of legacy space ISR systems.

\section{Background}

\section{Space Based ISR}

Satellites conducing ISR missions from the safety and impunity of Earth orbits is not a new concept. ISR satellites often referred to as Spy Satellites in the popular media, have circled the globe now for nearly five decades. Though the capabilities and the payloads of ISR satellite systems have changed over the years, nationally backed orbital bodies have been consistently gathering intelligence since the inception of the space race. Access to space has, until recently, been prohibitively expensive and technologically intensive allowing only the most wealthy and 
developed nations access to the seas of the $21^{\text {st }}$ century. With the advent of small, cheaper, faster microprocessors and more efficient launch vehicles, the final frontier is now accessible to nearly any group or government.

\section{Scales of Satellites}

Satellites can be broadly categorized into six basic classes based on the mass of the orbital body. Table 1 shows the classification of satellites by mass. Satellites, in the traditional sense, have tremendous masses and require apocalyptic amounts of thrust from launch vehicles to reach their orbital tracks. The Hubble Space Telescope, for example, weighs approximately 11,100 kg (12 tons) and is a large as a school bus. ${ }^{1}$ Satellites of this size and mass are monolithic in nature. Nearly every aspect of Large-class satellites is massive. Large-class satellites have corresponding large timelines, costs, size of launch vehicles, array of ground station support systems, and servicing mission requirements. Large Satellites are typically fielded by large technological and scientific economies of sovereign states like the United States, Russia, and China. Though more conglomerates like the European Union are entering into the Large Satellite arena, costs and capabilities preclude smaller countries from fielding large satellites. The tremendous capabilities of Monolithic Large Satellite programs come at a tremendous cost and nearly untenable timelines. The Hubble Space telescope spent 20 years in development $(1970-1990)$ and the Eurpoean Space Agency's MetOp Satellite was launched at a cost of $\$ 3.59 B$ (of which roughly $1 / 2$ was spent on the delivery vehicle due to the satellite's size and to which $\$ 11.2 \mathrm{M}$ was added due to a one month delay in production). ${ }^{2,3}$ 


\begin{tabular}{|c|c|c|}
\hline \multicolumn{2}{|c|}{ Satellite Class } & Mass \\
\hline Large Satellites & (Observatory, etc) & $>1000 \mathrm{~kg}$ \\
\hline MiniSats & \multirow{4}{*}{ SmallSat Class } & $100-1000 \mathrm{~kg}$ \\
\hline MicroSats & & $10-100$ kg \\
\hline NanoSats & & $1-10 \mathrm{~kg}$ \\
\hline PicoSats & & $.1-1 \mathrm{~kg}$ \\
\hline FemtoSats & & $1-100 \mathrm{~g}$ \\
\hline
\end{tabular}

Table 1: Satellite Classification by mass ${ }^{4}$

Satellites that fall short of the $1000 \mathrm{~kg}$ mark are sub-categorized into the SmallSat family. SmallSats range from $999 \mathrm{~kg}$ to .1kg and vary in capability, mission sets, and proliferation. Nations up-and-coming in space find it easier to enter the space market with SmallSats since they are generally cheaper to develop and field, and have a congruently smaller requirement for launch vehicles.

\section{Strength of the SmallSat}

Technology is, for the foreseeable future, inexorably tied to a engineering concept known as More's Law. This computer-engineering theorem dictates that the physical number of transistors able to be installed on an integrated circuit doubles roughly every 18 to 24 months. ${ }^{5}$ The continual miniaturizing of computing hardware with an inversely increase in capability from the same components have allowed SmallSats to stay competitive with the state-of-the-art rather the state-of-the-day. This is accomplished by shortening the production timeline of SmallSats to the point of designing, developing, and launching the satellites within a 24 month period. Whereas monolithic satellites often require decades of research and development, thus capturing state-ofthe-day technologies early on in development and carrying the legacy components forward through production, SmallSats have the capability of keeping inside the Moore's Law technology loop. A recent example of this is the Hubble Space Telescope, which was developed in the early 1970s through the mid-1980s. The Hubble's computer processors and sensors were, thanks to 
Moore's Law, roughly 20 times slower and less capable at the moment of launch in 1990 than other computer components on the market. ${ }^{6}$

SmallSats, though generally less capable than monolithic satellite programs, are able to significantly thin capability gaps between the two classes by keeping multiple generations of software and hardware ahead of even the most cutting-edge Large-class satellites. Monolithic satellite programs often undergo decades of design and production as a result of coupling the overtasking of a single orbital vehicle with too many missions with intense aversion to risk. To marginalize risk to mission, satellite designers routinely design dual (and often triple) redundant systems to ensure the vehicle's payload can accomplish the assigned mission - after all the failure of the orbital vehicle presents a single point of failure for the array of mission components present on the satellite. The presence of dual and triple redundant systems drives up costs and timelines of the satellites.

"The current approach... to designing space systems for robustness to uncertainty is the key to the sharply escalating costs and development timelines facing the space industry." ${ }^{7}$ In essence, monolithic satellites are economically and physically large because the single point of failure inherent in a monolithic systems drive the perceived need for redundant systems to ensure mission success, which adds weight, which makes them expensive to launch, which adds to the want for more redundant systems to ensure their success, and so on. Space program managers need to break the current paradigm of cramming multiple mission payloads into a single orbital vehicle. Small-class satellites offer a viable alternative to monolithic programs. SmallSats can be tailor made to conduct a single mission with significant capability thanks to advancements in computing power and software design. A single launch vehicle payload of multiple SmallSats could offer orbits of numerous satellites each with a unique or complimentary mission sets. ${ }^{8}$ This 
disperses the risk monolithic satellites routinely face. The failure of one satellite in a constellation of satellites doesn't necessarily equal the failure of the entire constellation. In essence, space program managers can break up the multiple missions of monolithic systems into a variety of smaller single focus satellites to drive down risk, cost, size, and weight.

\section{CubeSat}

CubeSats, are a specific type of PicoSats, and fall under the SmallSat family of Satellites. The term CubeSat is synonymous for both a type of and a sizing standard for SmallSats. To be classified as a CubeSat, the orbital body must be a $10 \times 10 \times 10 \mathrm{~cm}$ cube weighing no more than $1 \mathrm{~kg}^{9}$. This represents a 1 unit, or $1 \mathrm{u}$, CubeSat. A 2 unit CubeSat would measure 10 x 10 x $20 \mathrm{~cm}$ weighing $2 \mathrm{~kg}$, and a $3 \mathrm{u}$ CubeSat would measure 10 x 10 x $30 \mathrm{~cm}$ weighing $3 \mathrm{~kg}$, and so on. ${ }^{10}$ The standardization of CubeSats provides a number of complimentary benefits to space operations. Standard sizes and weights allow launch vehicle planners to easily incorporate CubeSats into a pre-existing launches. Launch providers regularly have extra room left in the delivery compartment of a rocket designed to deliver a large primary payload. This extra room and lift capacity presents potential added revenue and launch providers are eager to sell this extra room and delivery capability. CubeSats small size and standard measurements are a perfect fit for launch providers looking to maximize profit and utility.

As a tangential benefit to easily incorporating CubeSats into pre-existing launches, engineers can continually change the CubeSat's internal payload until quite late in the program timeline. The launch providers need only be concerned with the number of CubeSats a customer wishes to launch. ${ }^{11}$ The contents of the CubeSats are generally irrelevant to the launch provider as the size and weight standards ensure that the deployment of the satellites won't interfere with the large, often expensive, primary payload of the launch vehicle. 
Small standardized CubeSats can be easily fit to work around existing large primary payloads in delivery vehicles. Ease of fit and exponentially smaller sizes when compared to traditional satellite systems allow launch planners and satellite operators more opportunities to launch CubeSats and therefore more flexible to respond to changing mission sets and requirements. ${ }^{12}$ "Missing a launch entirely is not a huge problem, as you can simply book the next available flight - a very different situation to the one that faces engineers working on the primary [Large Satellite] payload, where even delays of a few weeks cost into the millions."13

\section{Current Coverage / Demand Disconnects}

So why does all this matter to US Special Operations Command? Prior to the $21^{\text {st }}$ century, a SmallSat was typically relevant only to hobbyists. Slow computing power and relatively large, heavy components prevented SmallSats from doing much more than answering Earth based beacons with basic waveform signals. Faster, lighter, and more capable components have vaulted SmallSats into quickly becoming operationally relevant to a high-end user like USSOCOM.

Nearly every aspect of SOCOM's activities require persistent and reliable overhead ISR, specifically those assets that produce imagery intelligence (IMINT) products. As the Combatant Command responsible for planning and executing all of America's counter terrorism activities, USSOCOM levies a tremendous requirement on all varieties of ISR platforms. If it flies and has a camera or sensor on it, USSOCOM is probably interested in what it can provide for the command. Special operators aren't the only game in town though, and USSOCOM finds itself continually in competition with all of the other Department of Defense (DoD) and US Government (USG) agencies vying for access to limited air breathing and space based ISR assets. As of 2015, USSOCOM controls of approximately $35 \%$ of all U.S. air-breathing ISR orbits world-wide. ${ }^{14}$ One combatant command putting such a demand on the ISR capability of 
the United States routinely leads to allocation friction between organizations. America's space based ISR platforms are often parsed into minute-by-minute allocation for the myriad of USG requests, and precious orbits of MQ-1 Predator and MQ-9 Reaper Remotely Piloted Aircraft (RPA) are fought over daily by Combatant Commander's staffs. With SOF controlling over a third of all US ISR orbits throughout the globe, and no foreseeable drawdown in operations or intelligence requirements, space based ISR could provide SOCOM an avenue to expand SOF's information networks without impinging on other joint operations.

SOF arguably has such a unique mission that simply jockeying for position to share RPA orbits or Satellite time blocks can create gaps in coverage requirements. USSOCOM's counter terrorism mission requires terrorists to be found, fixed, and finished when they are typically hiding, moving, and guarded. Whereas conventional forces use ISR to develop a literal picture of the battle space, Special Operations Forces (SOF) use ISR to track people over the course of days and weeks. The finding aspect SOF's find-fix-finish process is often the most difficult, time consuming, and volatile. To develop the fidelity necessary to conduct a strike against a high value target, an individual's the pattern of life must be established. This means a SOF strike team has to use overhead air breathing ISR to literally track a targeted individual with no gaps in coverage. A break in coverage of just one minute could result in mission abort. With some high value targets taking up to 600 hours of persistent ISR to track, a break in coverage could mean a massive waste in time and energy. ${ }^{15}$

Operations that require more than 500 hours of coverage in order to gain the proper fidelity on an individual's pattern of life are more frequent than not. Strikes of this magnitude typically results in a kinetic kill or a capture, and thus places a significant burden on ISR to provide high levels of fidelity and assurances required for such an operation to gain approvals. Operations 
including man-hunting of terrorists often takes place in access denied areas of the world where simply putting a MQ-1 or MQ-9 in orbit for days on end is neither possible nor feasible. USSOCOM needs an organic space based ISR capability to fill the need of persistent ISR in politically sensitive or denied areas of the world in order maintain it's decision making edge and operational supremacy over America’s enemies.

\section{Development of Organic SOF Space ISR Capabilities}

USSOCOM competes with the six Geographic Combatant Commanders (GCCs) for ISR orbits on a nearly day-to-day basis. While USSOCOM swings a very big bat in the ISR allocation discussion, there may be ways to augment and supplement the command's need to have a persistent perch over the enemies of the United States. Finding, fixing, following, and finishing the nation's enemies is SOCOM's business. While air-breathing ISR is very capable of doing this in specific venues, "It is not enough to deliver a given capability - it must be delivered with some degree of robustness in the face of various sources of risk or uncertainty." ${ }^{" 16}$ Airborne overhead ISR has one major capability gap: anti-access and access denied (A2AD) environments. The United States does not have the political will, for example, to park an MQ-9 remotely piloted aircraft over North Korea for weeks on end. Satellites, on the other hand, are a different story. The United States, along with every other developed nation on Earth, has leveraged the impunity of space to gather on otherwise A2AD areas of the globe like North Korea, China, Iran, and Russia.

\section{Use of SmallSats to Conduct ISR}

USSOCOM has already taken preliminary steps toward establishing an organic space based ISR capability with the launch of the Prometheus satellite constellation. SOCOM launched the Prometheus CubeSat constellation in 2013 as a technology demonstration aimed at exploring 
ways to fill current SOF communication and surveillance gaps. ${ }^{17}$ In all, eight CubeSats were successfully launched by a group of operators with little to no prior space experience for the cost of approximately $\$ 25,000$ per satellite. Communication with the constellation was established upon the satellites' primary orbit and continued though the vehicle's service life up to the point when the satellites re-entered the Earth's atmosphere thus proving two main points. One satellites can be launched by non-space savvy operators, and two - satellites can be launched cheaply and quickly if the means and necessity exist. ${ }^{18}$ But a technology demonstrator isn't where USSOCOM's capability to field an organic space based ISR system ends. Enter Kestrel Eye, the US Army's Space Missile Defense Command's (USASMDC) SmallSat sized, IMINT surveillance and reconnaissance satellite concept. ${ }^{19}$

"The Kestral [sic] Eye program will extend the Unmanned Aerial Vehicle (UAV) paradigm into space: a dramatically lower unit cost and proliferated numbers of satellites enabling the system to be dedicated to and operated by Warfighters. ${ }^{20}$ USASMDC's Kestrel Eye program aims to leverage the increasing capability of SmallSats into optical surveillance platforms small enough and proliferated enough to provide near instantaneous intelligence data to the tactical ground user directly. Essentially the Army wants a satellite constellation that allows the ground user access to space based visual spectrum imagery intelligence in the same manner ground forces are using tactical unmanned aerial vehicles (UAVs). USASMDC's concept of usercontrolled user-interfaced satellites isn't as far off as one may think. Using the Rayleigh Criterion (minimum angular separation for two objects to be resolved when viewed through a circular aperture) a SmallSat in a low earth orbit of $125 \mathrm{~km}$ above the surface would need only a $10 \mathrm{~cm}$ aperture to give viewers on Earth a $.75 \mathrm{~m}$ resolution. ${ }^{21}$ Such low earth orbits are difficult to maintain as the closer to Earth a satellite gets, the more the planet's atmosphere influences an 
orbiting body (drag) thus requiring more fuel to keep the satellite in orbit. A CubeSat's $10 \mathrm{~cm}$ aperture in a more comfortable perch of $160 \mathrm{~km}$ above the surface of the Earth would still yield an impressive $1 \mathrm{~m}$ image resolution. See Table 2.

\begin{tabular}{|c|c|c|c|c|c|}
\hline INCHES & METERS & NANO METERS & METERS & METERS & FEET \\
\hline $\begin{array}{c}\text { Diameter of } \\
\text { Aperature (D) }\end{array}$ & $\begin{array}{c}\text { Diameter of } \\
\text { Aperature (D) }\end{array}$ & $\begin{array}{c}\text { Light Wavelength } \\
(\lambda)\end{array}$ & $\begin{array}{l}\text { Altitude } \\
\text { (h) }\end{array}$ & $\begin{array}{c}\text { Resolution } \\
\text { (d) }\end{array}$ & $\begin{array}{l}\text { Resolution } \\
\text { (d) }\end{array}$ \\
\hline 4.00 & 0.10 & $5.00 \mathrm{E}-07$ & 125,000 & 0.75 & 2.46 \\
\hline 4.80 & 0.12 & $5.00 \mathrm{E}-07$ & 150,000 & 0.75 & 2.46 \\
\hline 5.12 & 0.13 & $5.00 \mathrm{E}-07$ & 160,000 & 0.75 & 2.46 \\
\hline 5.92 & 0.15 & $5.00 \mathrm{E}-07$ & 185,000 & 0.75 & 2.46 \\
\hline
\end{tabular}

\begin{tabular}{|c|c|c|c|c|c|}
\hline INCHES & METERS & NANO METERS & METERS & METERS & FEET \\
\hline $\begin{array}{c}\text { Diameter of } \\
\text { Aperature (D) }\end{array}$ & $\begin{array}{c}\text { Diameter of } \\
\text { Aperature (D) }\end{array}$ & $\begin{array}{c}\text { Light Wavelength } \\
\text { (入) }\end{array}$ & $\begin{array}{l}\text { Altitude } \\
\text { (h) }\end{array}$ & $\begin{array}{l}\text { Resolution } \\
\text { (d) }\end{array}$ & $\begin{array}{l}\text { Resolution } \\
\text { (d) }\end{array}$ \\
\hline 3.00 & 0.08 & $5.00 \mathrm{E}-07$ & 125,000 & 1.00 & 3.28 \\
\hline 3.60 & 0.09 & $5.00 \mathrm{E}-07$ & 150,000 & 1.00 & 3.28 \\
\hline 3.84 & 0.10 & $5.00 \mathrm{E}-07$ & 160,000 & 1.00 & 3.28 \\
\hline 4.44 & 0.11 & $5.00 \mathrm{E}-07$ & 185,000 & 1.00 & 3.28 \\
\hline
\end{tabular}

Table 2: Optical resolution as a result of aperture size at a given altitude above the Earth

The one obvious shortfall to the Kestrel Eye concept is size constraints. Although theoretically possible, the aperture size of a satellite in low earth orbit would take up the vast majority of the CubeSat's payload leaving little room for required navigation, power, communication, or sensor packages. CubeSats whose entire payload is monopolized with an optical sensor and image processor aren't very useful to anyone beyond the common hobbyist. The images and data collected by the satellite must be quickly and easily transmitted to the end user on Earth. This poses a problem for CubeSats as the small size and weight of the satellites are their most useful traits. The mass constraint of a single CubeSat can be solved by fractionating, or spreading, the CubeSat's functions among a closely flying interconnected CubeSat constellation.

Fractionated satellite technologies applied on a CubeSat scale and coupled with advances in wireless transmission of power (witricity) could provide solutions necessary to make CubeSat scaled ISR constellations a reality. A fractionated CubeSat constellation is, in essence, a group of 
CubeSats flying in orbit together, interconnected with a communications link, with each satellite tasked to perform a specific role in support of the overall mission of the constellation. Figure 1 shows a hypothetical fractionated orbital constellation with four individual CubeSats acting as an imagery sensor, a central processor, a power supply, and a data link to a ground station. Compare a fractionated satellite to an Army Special Forces A-Team. The twelve member team functions as one unit with specialty skills fractionated among its members. The A-Team's 18A is the team leader (CubeSat processor), the 18B is the team's weapons sergeant (CubeSat imagery sensor), the $18 \mathrm{D}$ is the team's medical sergeant (CubeSat power supply), and the $18 \mathrm{E}$ is the team's communications sergeant (CubeSat data link). Just as the strength of an A-Team greater that the sum of its parts, the fractionated CubeSat constellation would more powerful than the aggregated strength of its components.

Fractionated satellite constellation architectures take monolithic satellites and divide the subsystems into individual satellites. The "functionality of a traditional 'monolithic' spacecraft is delivered by a cluster of wirelessly-interconnected modules capable of sharing their resources and utilizing resources found elsewhere in the cluster." 22 Not only does a fractionated satellite constellation enable the launch of small vehicles in a timely manner, it also diffuses the risk of total system failure among numerous satellites. If one satellite in a constellation were to fail, say for example the CubeSat containing the processor, that satellite could be de-orbited and replaced with a new CubeSat within a very short time frame (often within a month). CubeSat constellations would also drastically shorten development timelines as the entire constellation would not have to undergo design and re-design every time a component becomes obsolete or ends its life cycle. For example, the optical sensor could be state of the art, but the data link to Earth could be two generations old. Instead of redesigning the entire system around a new data 
link, the up-to-date components could be kept in orbit and older components de-orbited, and replaced in short order by CubeSats with more advanced payloads.

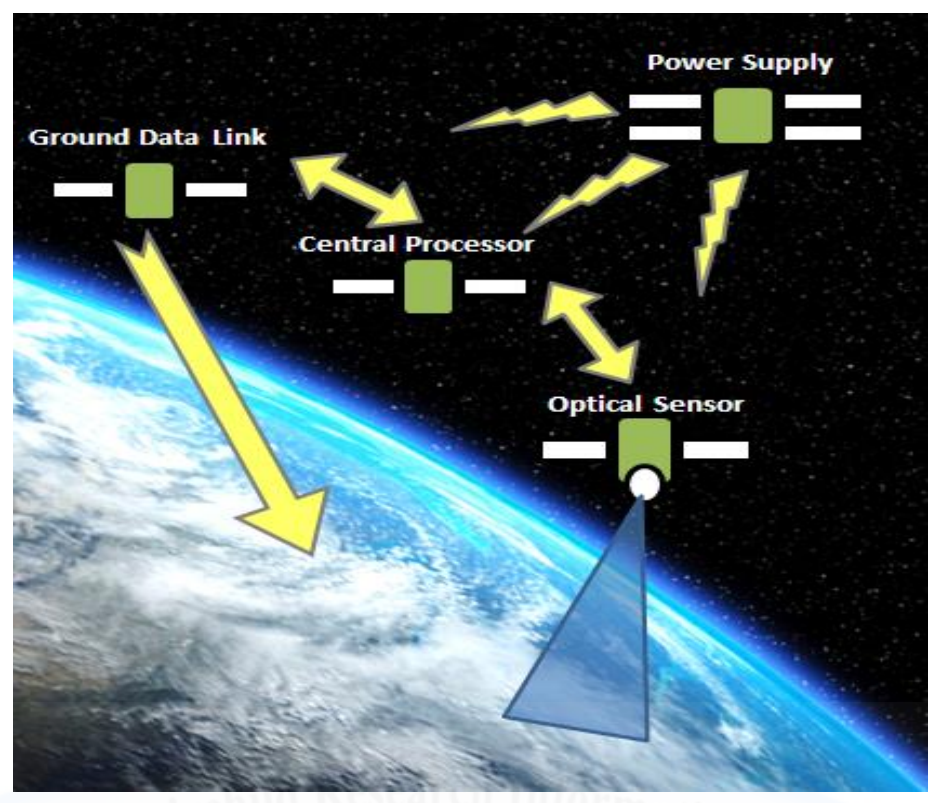

Figure 1: Theoretical Concept of a Fractionated Satellite Constellation

\section{Unique SOF Acquisition Capabilities}

USSOCOM is one of the few USG agencies that can realize the full potential of a CubeSat constellation. While all of the Geographic and Functional Combatant Commanders have to work through their respective service components in order to incorporate their unique capability requirements into large acquisition programs (aircraft, ships, tanks), USSOCOM does not. USSOCOM was granted special acquisition authority when it was created in 1987 . The Commander of USSOCOM has very similar acquisition capability as service chiefs and therefore can authorize and field SOF specific equipment quickly and effectively. USSOCOM's Science and Technology Directorate is uniquely capable of conducting feasibility assessments and determining the potential application the capabilities of unique systems like an ISR CubeSat fractionated constellation could offer USSOCOM. 


\section{Implementation of SOF ISR Constellation}

If SOCOM would venture into the space based ISR environment as a means to augment and supplement current ISR coverage, the command would have to address the operational deliverables of the satellite constellation with the scale and scope of the program underlying the requirements.

\section{Scale}

The cost of a CubeSat constellation would be controlled by the scope and vice-versa. In general though, the cost for a single CubeSat using mostly Commercial Off-The-Shelf (COTS) material ranges from $\$ 15,000$ - $\$ 35,000 .{ }^{23}$ Six constellations of ten fractionated CubeSats would cost approximately $\$ 5 \mathrm{M}$, which is $\$ 10 \mathrm{M}$ less than a single $\mathrm{MQ}-1$ Predator, and on par with fielding a new US Army SHADOW unmanned aerial system ${ }^{24,25}$. These constellations would provide the SOF user similar imagery products and coverage times as the MQ-1 while flying in uncontested space with relative impunity. A Kestrel Eye type fractionated constellation of CubeSats would also take numerous human operators out of the control loop in the same manner that tactical unmanned aerial vehicles are directly controlled by tactical ground units. ${ }^{26}$ The scale of a CubeSat program would also vary greatly depending on the constellation's mission. The need for high revisit rates (the number of times per day a satellite flies over the same target on Earth) would drive the need for more constellations in a given orbit and thus drive costs up.

\section{Scope}

The required scope of the program would directly drive a CubeSat constellation's capabilities. Persistent coverage with relatively short time from gathering to presentation to user would yield a very specific constellation composition while multi-spectral sensing coverage with long dwell time over target would yield a categorically different CubeSat constellation. In either 
case, the architecture must ensure the collected intelligence can be presented to user in a simple to manipulate fashion. Like the Army's Kestrel Eye, if SOCOM were to field a space based ISR constellation, it would have to give the user the ability receive the space based ISR imagery directly from the imagery sensor in orbit.

\section{Recommendations}

USSOCOM is a command of many dimensions. Though there are some nascent stages of space operations within the command, organically fielded fractionated constellations of CubeSats using existing SOF lines of accounting would significantly increase the SOF warfighter's situational awareness of the battlefield. CubeSats can provide the SOF warrior relatively cheap and flexible ISR options. A CubeSat constellation has the potential to provide tactical UAV like capabilities with the added advantage of operating in an uncontested environment (Space). Where current gaps in coverage exist over nations and states that would be considered politically denied areas of the world for air-breathing ISR overflight, CubeSats provide the warfighter with the marked advantage to be perched in space and still have the tactical control over ISR required to fill mission requirements. USSOCOM could realize tangible results from this program by allowing its forces access to a warfighting domain that was once considered only accessible through time sharing national space assets through other governmental agencies.

Along with considering what types of capabilities and coverage an space based ISR constellation would provide the warfighter, SOCOM need also address where the personnel and personnel and equipment fit into its current force structure. Traditionally, space ISR assets are, like air assets, spread throughout the joint force with centralized command and control being retained by a single organization and the effects being divided through theater or force 
allocation. The addition of an organic space based ISR capability to the SOCOM repertoire of force support does not, and should not follow this traditional model, however. A space based ISR platform such as a fractionated CubeSat constellation should be task-organized under specific operations. In this construct, the TSOC commander should have a space office within his staff. By his command, the space office would allocate use of the constellation to a specific operation. Say, for example, there was a SOF team attempting to determine the pattern of life of a known terrorist leader in Country X. Country X won't allow persistent overflight of unmanned aerial vehicles, so the TSOC responsible for Country X could allocate dedicated use of a CubeSat constellation to the SOF team for the duration of the pattern of life establishment. This is just one of many hypothetical structures the addition of an organic space based ISR constellation could fall under.

In any case, SOCOM needs to understand and hold fast to the premise that in fielding an organic space capability, the vehicle and the method of intelligence delivery has to maintain an operational posture. Surrendering control over any aspect of the program to an organization outside of SOCOM will immediately negate this tactical necessity. SOCOM needs to also accept that a fractionated CubeSat ISR constellation would not fall under traditional intelligence or communication directorates. Just as SOF aviation wouldn't fall under J2 or J6, neither should SOF space. Finally, if SOCOM is to venture into the space based ISR business, it needs spacesavvy SOF personnel, but none with institutional biases or monolithic space program tendencies (the successful launch of the eight-satellite Prometheus constellation was completed by nonspace SOF operators). Keeping the program SOF will prevent the program from veering too much into traditional space force realms. 


\section{Conclusion}

USSOCOM has an incredibly demanding mission that requires a significant amount of manpower, equipment, and capability support from a variety of different warfighting domains. In executing its mission as the nation's lead counterterrorism combatant command, its forces will continue to put a strain on the nation's air-breathing ISR capability. In order to effectively track and eliminate our most dangerous adversaries, USSOCOM needs to consider fielding the capability to procure, configure, launch, and control its own constellation of space based ISR satellites. To field its own space based ISR capability, USSOCOM should seriously consider using a fractionated constellation of CubeSats as the architecture to support its uniquely sensitive and time critical mission sets. CubeSats could provide the command with an organic cheap, flexible, customizable satellite based ISR capability that could augment and supplement its current intelligence requirements on the battlefields throughout the Earth. 


\section{Notes}

(All notes appear in shortened form. For full details, see the appropriate entry in the bibliography)

1 NASA, Hubble Space Telescope

2 Edwards, "The Cubed Route", 68

3 Kramer and Cracknell, "An overviw of small satellites in remote sensing", 4287

4 Ibid., 4290

5 Moore, "Cramming More Components onto Integrated Circuits", 82-85

6 Kramer and Cracknell, "An overviw of small satellites in remote sensing", 4287

7 Brown and Eremenko, The Value Prposition For Fractionated Space Architectures, 2

8 Kramer and Cracknell, "An overviw of small satellites in remote sensing", 4291

9 Thomas, Prototype Development and Dynamic Characterization of Deployable CubeSat Booms, 13

${ }^{10}$ Mattox, The Space Review

11 Thomas, Prototype Development and Dynamic Characterization of Deployable CubeSat Booms, 17

${ }^{12}$ Kramer and Cracknell, "An overviw of small satellites in remote sensing", 4291

${ }^{13}$ Edwards, "The Cubed Route", 70

${ }^{14}$ USSOCOM Intelligence Officer

${ }^{15}$ Goodman, "ISR Now Synonymous With Operations", 19

${ }^{16}$ Brown and Eremenko, The Value Prposition For Fractionated Space Architectures, 1

${ }^{17}$ Mattox, The Space Review

${ }^{18}$ Mattox, The Space Review

${ }^{19}$ US Army Space Missile Defense Command,"Kestrel Eye Visible Imagery Nanosatellite Technology Demonstration"

${ }^{20}$ Grigsby, Satellite Capabilities Mapping - Utilizing Small Satellites, 18-20

${ }^{21}$ Luttermoser, PHYS-2020: General Physics II Course Lecture Notes Section XIV, XIV-7

${ }^{22}$ Defense Advanced Research Projects Agency, DARPA Tactical Technology System F6

${ }^{23}$ Pumpkin Incorporated, "Pumpkin Satellite Parts Price List"

${ }^{24}$ USSOCOM Intelligence Officer

${ }^{25}$ Office of the Under Secretary of Defense (Comptroller) / Chief Financial Officer, Fiscal Year 2015 Budget Requst: Program Acquisition Cost By Weapon System

${ }^{26}$ US Army Space Missile Defense Command,"Kestrel Eye Visible Imagery Nanosatellite Technology Demonstration" 


\section{Bibliography}

Ajey, Kumar R., H. R. Gayathri, Gowda R. Bette, and B. Yashwanth. "WiTricity: Wireless Power Transfer By Non-radiative Method." Internatinoal Journal of Engineering Trends and Technology 11, no. 6 (May 2014): 290-295.

Brown, Owen, and Paul Eremenko. The Value Prposition For Fractionated Space Architectures. Topic Research, Fairfax, VA: Defense Advanced Research Projects Agency, 2008.

Defense Advanced Research Projects Agency. DARPA Tactical Technology System F6. n.d. http://www.darpa.mil/our_work/tto/programs/system_f6.aspx (accessed 03 01, 2015).

Edwards, Chris. "The Cubed Route." Engineering \& Technology, 2013: 68-72.

Goodman, G. "ISR Now Synonymous With Operations." The Journal of Electronic Defense 30, no. 7 (July 2007): 19-20.

Grigsby, David. Satellite Capabilities Mapping - Utilizing Small Satellites. MS Thesis, WrightPatterson Air Force Base, Ohio: Air Force Institute of Technology, 2010.

Keller, John. Military \& Aerospace Electronics. December 2, 2012.

http://www.militaryaerospace.com/articles/2012/12/raytheon-darpa-seeme.html (accessed February 25, 2015).

Kramer, Herbert J., and Aurthur P. Cracknell. "An overviw of small satellites in remote sensing." International Journal of Remote Sensing 29, no. 15 (August 2008): 4285-4292.

Luttermoser, Donald G. "PHYS-2020: General Physics II Course Lecture Notes Section XIV." Class Instruction Notes, Physics, East Tennessee University, Johnson City, TN, 2012. Mattox, Ethan W. The Space Review. April 14, 2014. http://www.thespacereview.com/article/2491/1 (accessed February 11, 2015). 
AU/ACSC/HELMS/AY15

Moore, Gordon E. "Cramming More Components onto Integrated Circuits." 86, no. 1 (1998): 8285.

NASA. NASA Hubble Space Telescope. n.d. http://www.nasa.gov/mission_pages/hubble/story/ (accessed February 25, 2015).

Office of the Under Secretary of Defense (Comptroller) / Chief Financial Officer. US DoD Fiscal Year 2015 Budget Requst: Program Acquisition Cost By Weapon System. Budget, Washington D.C.: US Department of Defense, 2015.

Pumpkin Incorporated. "Pumpkin Satellite Parts Price List." Electronic Parts Pricing, San Fransisco, CA, 2015.

Senatore, Patrick, Andrew Klesh, Thomas Zurbuchen, Darren McKague, and James Cutler. "Concept, Design, and Prototyping of XSAS: A High Power Extendable Solar Array for CubeSat Applications." 40th Aerospace Mechanisms Symposium. NASA Kennedy Space Center: NASA/CP, 2014. 431-444.

Thomas, Grant M. Prototype Development and Dynamic Characterization of Deployable CubeSat Booms. MS Thesis, Wright-Patterson Air Force Base, OH: Air Force Institute of Technology, 2010.

US Army Space Missile Defense Command. "Kestrel Eye Visible Imagery Nanosatellite Technology Demonstration." US Army SMDC. February 16, 2013. http://web.archive.org/web/20130216142346/http://www.smdc.army.mil/FactSheets/Kest relEye.pdf (accessed February 11, 2015).

USSOCOM Intelligence Officer, interview by Maj Christian Helms. ACSC ISR Elective Day 10 (March 25, 2015). 